\title{
WEAKLY MIXING TRANSFORMATIONS WHICH ARE NOT STRONGLY MIXING
}

\author{
R. V. CHACON $^{1}$
}

1. Introduction. The purpose of this note is to give a general method for constructing transformations which are weakly but not strongly mixing. The method will be given in a simple example from which its general features are clear. Applications of the method are given in [1] and [2].

2. Preliminaries. The examples will be of an invertible measure preserving transformation of the unit interval (equipped with the usual Lebesgue sets and Lebesgue measure).

The transformation will be constructed in a sequence of steps, each step enlarging the domain of definition of the transformation. The construction utilizes a geometric approach described in [1] which consists of mapping subintervals of the same length linearly onto each other, and of representing this geometrically by a figure in which each interval is placed below its image. Thus, if $I^{1}, \cdots, I^{n}$ are pairwise disjoint subintervals of the unit interval, if they have the same length, and if we define $\tau$ on $\bigcup_{k=1}^{n-1} I^{k}$ by mapping $I^{k}$ linearly onto $I^{k+1}, k=1, \cdots, n-1$ (and leave $\tau$ undefined elsewhere), the geometric figure that corresponds to this map consists of the intervals $I^{1}, \cdots, I^{n}$ arranged in a stack with $I^{1}$ on the bottom and $I^{n}$ on the top, and with the remaining intervals arranged in order between them, so that each point is located below its image. The action of the transformation can thus be regarded as an upward flow through the stack which ends when the top layer is reached, since the transformation is undefined there. Note that we have not assumed that the union of $I^{1}, \cdots, I^{n}$ is the unit interval.

The main advantage of the geometric approach is that many properties of transformations become much clearer when viewed in this way. Of course, the geometric figure associated with each stage of the definition is not necessary for the definition of the transformation nor for the proof of its properties. It serves simply as an aid in understanding the construction.

3. The main theorem. Let $(X, F, \mu)$ be the unit interval, the Lebesgue sets, and Lebesgue measure.

Received by the editors January 10, 1969.

${ }^{1}$ Research supported in part by NSF grant GP-7490. 
TheOREM. There exists an ergodic, invertible measure preserving transformation $\sigma$ which is weakly but not strongly mixing.

Proof. As indicated, the transformation will be constructed inductively. After the transformation is constructed, we will prove that it is not strongly mixing using a direct argument, and that it is weakly mixing by showing that its sole eigenvalue is the constant equal to one.

Step 1. We divide the unit interval into three pairwise disjoint and consecutive intervals which we denote by $R_{1}, I_{1}^{1}, I_{1}^{2}$, where $I_{1}^{1}$ and $I_{1}^{2}$ have the same length, and define $\tau_{1}$ by mappii $g I_{1}^{1}$ linearly onto $I_{1}^{2}$. The geometric figure that corresponds to $\tau_{1}$ cunsists of the stack composed of the interval $R_{1}$ with nothing above or below it, since $\tau$ is undefined on $R_{1}$, and since $R_{1}$ does not contain the image of any point under $\tau$, and next to this singleton stack, the stack composed of $I_{1}^{1}$ and $I_{1}^{2}$ with $I_{1}^{1}$ on the bottom and $I_{1}^{2}$ on the top. The length of $R_{1}$ and the common length of $I_{1}^{1}$ and $I_{1}^{2}$ at the moment satisfy only the condition that $\mu\left(R_{1}\right)+2 \mu\left(I_{1}^{1}\right)=1$, and will be further specified later.

Step $n$. We suppose that the unit interval is partitioned into $p(n)+1$ intervals, $\left\{R_{n}, I_{n}^{1}, \cdots, I_{n}^{p(n)}\right\}$ where the intervals $I_{n}^{1}, \cdots, I_{n}^{p(n)}$ have the same length, and where $\mu\left(R_{n}\right)+p(n) \mu\left(I_{n}^{1}\right)=1$, and where $\tau_{n}$ is defined by mapping $I_{n}^{k}$ linearly onto $I_{n}^{k+1}, k=1, \cdots, p(n)-1$. The geometric figure that corresponds to $\tau_{n}$ consists of the stack composed of the interval $R_{n}$ with nothing above or below it, and next to this singleton stack, the stack composed of $I_{n}^{1}, \cdots, I_{n}^{p(n)}$ arranged in order, with $I_{n}^{1}$ on the bottom and $I_{n}^{p(n)}$ on the top. $\tau_{n}$ is defined on $\bigcup_{k=1}^{p(n)-1} I_{n}^{k}$ and is undefined elsewhere. We now form the partition $\left\{R_{n+1}, I_{n+1}^{1}, \cdots, I_{n+1}^{p(n+1)}\right\}$ as follows: each interval $I_{n}^{\mathbf{k}}, k=1, \cdots$, $p(n)$, is written as the union of two consecutive intervals of the same length, $I_{n}^{k}=I_{n, 1}^{k}+I_{n, 2}^{k}$, and $R_{n}$ is written as the union of two consecutive intervals, $R_{n}=A_{n+1}+B_{n+1}$, where the length of $B_{n+1}$ is equal to the common length of the $I_{n, j}^{k}, k=1, \cdots, p(n), j=1,2$. The partition $\left\{R_{n+1}, I_{n+1}^{1}, \cdots, I_{n+1}^{p(n+1)}\right\}$ is now defined by setting $R_{n+1}=A_{n+1}$, $I_{n+1}^{k}=I_{n, 1}^{k}, \quad k=1, \cdots, p(n), \quad I_{n+1}^{p(n)+k}=I_{n, 2}^{k}, \quad k=1, \cdots, p(n), \quad$ and $I_{n+1}^{2 p(n)+1}=B_{n+1}$, so that $p(n+1)=2 p(n)+1$.

Geometrically, this corresponds to cutting an interval from the right side of $R_{n}$ of length equal to half the length of the $I_{n}^{\mathbf{k}}, k=1, \cdots$, $p(n)$, placing this interval over the right-hand side of $I_{n}^{p(n)}$ and then in cutting the stack $I_{n}^{k}, k=1, \cdots, p(n)$ down the middle, and placing the right-hand side (with the extra interval from $R_{n}$ on top) over the left-hand side. 
The transformation $\tau_{n+1}$ is now defined by mapping $I_{n+1}^{k}$ linearly onto $I_{n+1}^{k+1}, k=1, \cdots, p(n+1)-1$.

Properties of $\left\{\tau_{n}\right\}$. It is clear from the geometric interpretation that $\tau_{k+1}=\tau_{k}$ on the domain of definition of $\tau_{k}$, and that $\lim _{k \rightarrow \infty} \tau_{k}$ exists. We may also see from the geometric interpretation that the limit exists almost everywhere on the unit interval if the lengths of $R_{1}, I_{1}^{1}, I_{1}^{2}$ are chosen properly. A simple calculation tells us that the proper choice is $\mu\left(R_{1}\right)=\mu\left(I_{1}^{1}\right)=\mu\left(I_{1}^{2}\right)=1 / 3$, so that at the $n$th stage $\mu\left(R_{n}\right)=\mu\left(I_{n}^{k}\right), \quad k=1, \cdots, p(n)$, and $\mu\left(R_{n}\right)=1 /(p(n)+1), \quad p(n)=$ $2 p(n-1)+1, p(1)=2$.

Properties of $\sigma=\lim _{k \rightarrow \infty} \tau_{k}$. We first show that $\sigma$ is not strongly mixing. The property needed to see this is that $\sigma$ maps half of $I_{n}^{p(n)}$ into $I_{n}^{1}$, for each $n$, and that the class of sets $a_{n}$ which are unions of some of the intervals $\left\{I_{n}^{1}, \cdots, I_{n}^{p(n)}\right\}$ can be used to approximate any set $A \in \mathcal{F}$ in the sense that $\lim _{k \rightarrow \infty} \mu(A \Delta A(k))=0$ where $A(n)$ is the set of $\mathrm{Q}_{n}$ for which $\mu(A \Delta A(n))$ is a minimum (for $n$ fixed). A simple argument then shows that

$$
\mu\left(A \cap \sigma^{p(k)} A\right) \geqq \frac{1}{2} \mu(A)-\delta(k),
$$

where $\delta(k) \rightarrow 0$. This would contradict strong mixing, since it would imply that $\{\mu(A)\}^{2} \geqq(1 / 2) \mu(A)-\delta(k), \delta(k) \rightarrow 0$.

To see that $\sigma$ has no eigenvalues other than the constant one, the property needed is that $\sigma$ maps half of $I_{n}^{p(n)}$ into $I_{n}^{1}$, as is needed to show that $\sigma$ is not strongly mixing, and the additional property that one quarter of $I_{n}^{p(n)}$ is mapped into $I_{n}^{1}$ by $\sigma^{2}$. That one-quarter of $I_{n}^{p(n)}$ is mapped into $I_{n}^{1}$ by $\sigma^{2}$ can be seen by noting that half of $I_{n}^{p(n)}$ is mapped into $R_{n+1}$, and that half of this set is mapped into $I_{n+1}^{1}$, which is contained in $I_{n}^{1}$. We also need to know that the sets $Q_{n}$ approximate any set $A \in \mathcal{F}$, or what is the same thing, that any function measurable with respect to $f$ can be approximated by a function $f_{n}$ measurable with respect to $a_{n}$, and having support on $\bigcup_{k=1}^{p(n)} I_{n}^{k}$, in the sense that

$$
\lim _{n \rightarrow \infty} f_{n}=f
$$

in $L_{1}$.

We next show that $\sigma$ cannot have an eigenvalue other than one corresponding to an eigenfunction $f$ which is constant on one of the intervals $\left\{I_{n}^{1}, \cdots, I_{n}^{p(n)}\right\}$, for some $n$, say on the interval $I_{n}^{\boldsymbol{k}(0)}$, $I \leqq k(0) \leqq p(n)$. We have indicated that $\sigma$ maps $I_{n}^{\boldsymbol{k}}$ onto $I_{n}^{\boldsymbol{k}+1}$, $k=1, \cdots, p(n)-1$, that $\sigma$ maps half of $I_{n}^{p(n)}$ onto $I_{n}^{1}$ and that $\sigma^{2}$ maps a quarter of $I_{n}^{p(n)}$ onto $I_{n}^{1}$. This clearly implies that $\sigma^{p(n)}$ maps half 
of $I_{n}^{\mathbf{k}(0)}$ into $I_{n}^{\mathbf{k}(0)}$ and that $\sigma^{p(n)+1}$ maps a quarter of $I_{n}^{\boldsymbol{k}(0)}$ into $I_{n}^{\mathbf{k}(0)}$. If $f=\alpha$ on $I_{n}^{k(0)}$ and if it has eigenvalue $\lambda$, we would then have

$$
\begin{aligned}
f\left(\sigma^{-p(n)} x\right) & =\lambda^{p(n)} f(x) \\
f\left(\sigma^{-p(n)-1} x\right) & =\lambda^{p(n)+1} f(x) .
\end{aligned}
$$

If we let $x \in I_{n}^{k(0)} \cap \sigma^{p(n)} I_{n}^{\mathbf{k}(0)}$ and substitute in the first equation, we obtain

$$
\alpha=\lambda^{p(n)} \alpha .
$$

If we let $x \in I_{n}^{k(0)} \cap \sigma^{p(n)+1} I_{n}^{k(0)}$ and substitute in the second equation, we obtain

$$
\alpha=\lambda^{p(n)+1} \alpha .
$$

These two equations then imply that $\lambda=1$.

To see that $\sigma$ cannot have an eigenvalue other than one corresponding to a general eigenfunction (that is, other than those constant on some $\left.I_{n}^{k(0)}\right)$; note that for a fixed $\alpha \neq 0$ and each $\epsilon>0$, there is an $n$ and a $k(0)$ such that $|f-\alpha|<\epsilon$ for some constant $\alpha$ on a subset of $I_{n}^{\boldsymbol{k}(0)}$ of measure greater than $(1-\epsilon) \mu\left(I_{n}^{\boldsymbol{k}(0)}\right)$. If $\epsilon<1 / 4$, an argument similar to the previous one shows that

$$
\begin{aligned}
& \alpha+\delta_{1}=\lambda^{p(n)}\left(\alpha+\delta_{2}\right) \\
& \alpha+\delta_{3}=\lambda^{p(n)+1}\left(\alpha+\delta_{4}\right)
\end{aligned}
$$

where $\delta_{1}, \delta_{2}, \delta_{3}, \delta_{4}$ and $n$ depend on $\epsilon$, and where $\left|\delta_{i}\right|<\epsilon, i=1,2,3,4$. These equations imply that

$$
\lambda=\frac{\left(\alpha+\delta_{3}\right)\left(\alpha+\delta_{2}\right)}{\left(\alpha+\delta_{1}\right)\left(\alpha+\delta_{4}\right)}
$$

from which it follows that $\lambda=1$.

\section{BIBLIOGRAPHY}

1. R. V. Chacon, A geometric construction of measure preserving transformations, Proc. Fifth Berkley Sympos. Math. Statist. and Probability (Berkley, 1965/66), vol. II : Contributions to probability theory. Part 2, Univ. of California Press, Berkley, Calif., 1967, pp. 335-360.

2. - Approximation of transformations with continuous spectrum, Pacific J. Math. (to appear).

UNIVERSITY OF MiNNESOTA 